\title{
Inflation in a Web
}

\author{
Sheng $\mathrm{Li}^{a}$, Yang $\mathrm{Liu}^{a, b}$, Yun-Song Piao ${ }^{a}$ \\ ${ }^{a}$ College of Physical Sciences, Graduate School of Chinese Academy of Sciences, Beijing 100049, China and \\ ${ }^{b}$ Department of Physics, Shandong University, Jinan, 250100, China
}

\begin{abstract}
In a given path with multiple branches, in principle, it can be expected that there are some fork points, where one branch is bifurcated into different branches, or various branches converge into one or several branches. In this paper, it is showed that if there is a web formed by such branches in a given field space, in which each branch can be responsible for a period of slow roll inflation, a multiverse separated by domain wall network will come into being, some of which might corresponds to our observable universe. We discuss this scenario and show possible observations of a given observer at late time.
\end{abstract}

PACS numbers:

\section{INTRODUCTION}

Recently, the string landscape [1], 2] with large number of vacua has received increasing attentions. In the low energy limit, the landscape can be visualised as a complicated and rugged potential in a given field space with multiple dimensions. The large number of extrema in the landscape means there is an exponentially large number of paths for going down hill, some of which may be suitable candidates for inflation [3], [4], [5], [6].

In a randomly chosen downhill path, which generally has zigzags and turns as a result of the complexity of the landscape, there preferable be multiple stages of inflation, each of which is dominated by the effective potential respectively. The notion that inflation with multiple stages e.g. [7], [8], [9], 10], [11], [12] is appealing, since it can lead to a significant scale dependence of spectral index of curvature perturbation. However, this time we will not merely focus on a single downhill path as commonly investigated but on the overall configuration of the interveined paths. Specifically speaking, there should be certainly lots of fork points in a given path of field space, where one branch is bifurcated into different branches, or various branches converge into one or several branches ${ }^{1}$. In this sense, this corresponds that there is a "web" formed by connected branches in which each realistic path is consisted of a series of conjoint branches. Considering what if the inflation is driven by such a web, which is our purpose of the paper, might be interesting since conventionally the background of inflating universe is homogenous all along for slow rolling inflaton, however, here since the fork points inevitably alter the experience of different regions of inflating universe, such homogeneousity can be hardly preserved in whole space. Therefore, we need to check the global configuration of inflating universe under such a web.

When the effective inflaton field arrives at a given fork point (here the effective inflaton means the effective field

\footnotetext{
${ }^{1}$ where the word "stage" is replaced with "branch", which can be more visual for our purpose.
}

moving along certain branch of a given path in field space, which can be one or the synthesis of several fields) the inflating universe will turn into many independent regions separated by domain walls ${ }^{2}$. The probability that the effective inflaton enters into certain branch is determined by the characters of potential around the corresponding fork point and the history. After this splitting, the evolutions of different regions will be generally different as the effective inflaton in each new branch has different potentials. In this sense, each of different regions actually corresponds to a new universe, which can be stretched exponentially during its following inflation. When the effective inflaton meets the fork again, the separation will inevitably occur again. Thus it can be seen that the existence of such a web of branches will lead to a multiverse separated by domain wall network, especially, discriminate from multiverse scenario, this multiverse is complemented by the classical rolling of inflaton rather than the random walk by its large quantum fluctuation and therefore takes place at a relatively lower energy scale. We will discuss this scenario, and show possible observations of a given observer at late time, if our universe is just one of this multiverse system.

\section{DESCRIPTION OF SCENARIO}

We begin with a potential plotted in Fig.1, in which a simple web of branches is given, and each of these branches is assumed to be able to give a period of slow roll inflation. In general, when the scale of potential is sufficiently high the fluctuation of field can be expected to overwhelm its classical evolution. In this case, the effective inflaton field will be in stochastic walking with

\footnotetext{
2 Here, the fork point is slightly similar to the 'waterfall' point in hybrid inflation [13], also [14], 15], [16], [17]. The appearance of domain walls is a main problem of original hybrid inflation, which needs to be avoided, see [18], [19], 20]. Here, however, we identify the regions inside domain walls as different universes, which experience the slow roll inflation till the effective inflaton meets the fork point again or reheats.
} 


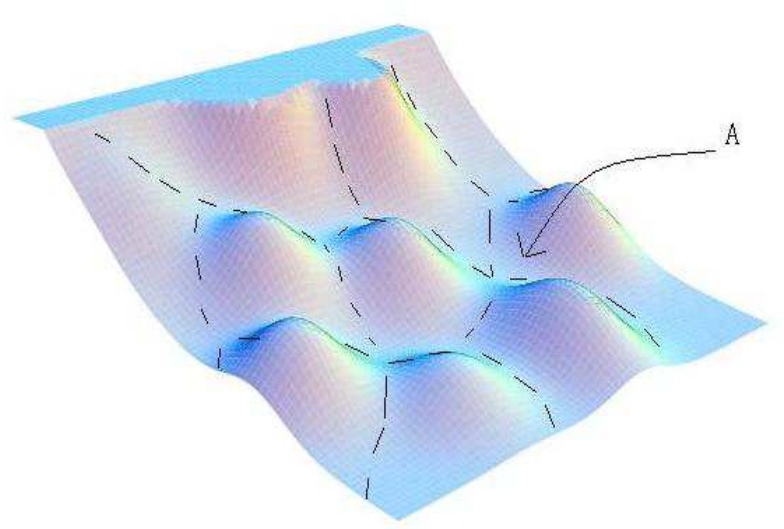

FIG. 1: The sketch of branches web. The dashed lines correspond to the branches, along which the effective inflaton rolls down. There are some fork points, where one branch is bifurcated into different branches, or various branches converge into one or several branches.

the step length $\frac{H_{\text {inf }}}{2 \pi}$, where $H_{\text {inf }}$ is the inflation scale. In unit of time $\frac{1}{H_{\text {inf }}}$, different regions with length scale $\frac{1}{H_{\text {inf }}}$ will has different energy density and thus denote different universes. This scenario has been called as eternal inflation 21, 22], and also 23], 24], which leads to an inflationary multiverse. In some universes of this inflating multiverse, the field will fluctuate up and the selfproduction of universes continues. While in some other universes the field will fluctuate downhill and into a regime where the classical evolution dominates and the slow roll inflation will appear. This eternal inflation in certain region could actually guarantee that the web which is downriver of it will eventually get throughout covered. In general, it is thought for the latter that a reheating will occur and then the corresponding universe will enter into an evolution of standard cosmology. Here, however, we will show that in a downhill path, due to the possible existence of some fork points, the case will not so simple.

When the effective inflaton rolls down along its branch ${ }^{3}$, it is bounded well in its orbit. While the effective inflaton arrives at the fork point, e.g.'A', its orbit will becomes an unstable ridge. The deviation of the extremum, which is induced by the fluctuation of field [25], 26], 227], 28], along other branches will bring the field off the ridge and make it roll down to the corresponding branch. In the momentum space, the fluctuation of field $\varphi$ is $\delta \varphi_{k} \simeq \frac{H_{\text {inf }}}{\sqrt{2 k^{3}}}$, which means in time interval $\frac{1}{H_{\text {inf }}}$, the fluctuation in coordinate space is given by $\frac{H_{\text {inf }}}{2 \pi_{1}}$, which occurs in different regions with length scale $\frac{1}{H_{\text {inf }}}$. The

3 Hereafter, for our purpose we will be constrained to the case that the fluctuation of field is not larger than its classical evolution. randomicity of fluctuation means that each different $\frac{1}{H_{\text {inf }}}$ region will be expected to enter into different branch. In principle, the number of new bifurcated branches at fork point should be large, since the dimension of field space is quite large. In general, different branches should have different potentials unless there is a large fine tunning,and the subsequent evolutions of different regions are generally different. In this sense, each of these independent regions actually corresponds to a new universe. These new universes are generally separated by domain walls ${ }^{4}$, which attributes to the complex structure of effective potential.

The number of different regions, or universes, which are separated by domain walls after 'A', is given by the efolding number of inflation along the branch before 'A'. The universe with initial length $\frac{1}{H_{\text {inf }}}$ will become $R \simeq \frac{1}{H_{\text {inf }}} e^{\mathcal{N}}$ after $\mathcal{N}$ efoldings. Thus the number of new universes after ' $\mathrm{A}$ ' is

$$
N_{\mathrm{A}}=\left(\frac{R}{\left(\frac{1}{H_{\text {inf }}}\right)}\right)^{3} \simeq e^{3 \mathcal{N}},
$$

which shows that the larger the efolding number is, the more the number of new universes after ' $A$ ' would be. The inflation in each new universe will be driven by the effective potential of corresponding new branch. When the effective inflaton along this branch meets the fork point again, a similar splitting process will appear. In principle, this separation can occur for all new universes, each of which undergoes an expansion driven by the inflaton along itself branch. In this way, with the lapse of time, a multiverse actually comes into being ${ }^{5}$.

In principle, it can be imagined that all paths could end at certain stable or metastable minimum, in which reheating might occur in some universes, lie with the potential in corresponding branch, and then these universes will begin the evolution of standard cosmology. The number of universes eventually entering into certain stable or metastable minimum can be estimated as

$$
N=\sum_{\text {all paths }}\left(\prod_{j} f_{i j} e^{3 \mathcal{N}_{i j}}\right),
$$

\footnotetext{
${ }^{4}$ However, if the number of new bifurcated branches at corresponding fork point is quite few, it is possible that there are lots of adjacent $\frac{1}{H_{\text {inf }}}$ regions which will enter into same branch. In this case, there will be not the domain walls between them.

${ }^{5}$ In practice, there are certainly some fork points, at which some branches converges into one branch. However, in this case, generally it can hardly expected that the regions dominated by these branches can be incorporated into one single universe, since the experiences of these regions are certainly different before their effective inflatons arrive at the fork point, unless there is a large fine tunning for the effective potentials of different branches. However, if there are only slight difference between the effective potentials of corresponding branches, the regions from different branches may be incorporated into one observable universe, which can have distinct features in its CMB power spectrum [29].
} 
where $f_{i j}$ denotes the probability entering the $i$ th branch at the $j$ th fork point, thus $f_{i j} \lesssim 1$ and $\sum_{i} f_{i j}=1$ are required, and $\mathcal{N}_{i j}$ is the efolding number of inflation in which the effective inflaton slow rolls along the $i$ th branch beginning at the $j$ th fork point. Here, different paths means that for each path, there is at least one branch, which is different from any other path. In principle, there can be lots of paths be able to arrive at the same minimum. Thus there is a sum for all paths, whose end point is at certain minimum. $f_{i j}$ is generally determined by the characters of corresponding fork point, e.g. the number of branches and the tilt of effective potential along different branches, and the evolutive history before it. Though $f_{i j} \lesssim 1$, generally $e^{\mathcal{N}_{i j}} \gg 1$, which can render $f_{i j} e^{\mathcal{N}_{i j}} \gtrsim 1$ in corresponding branch of given path. Thus $N$ can be generally quite large.

The time that the effective inflaton spends in each branch should be different, since the effective potential of each branch is generally different, and it can be estimated as follows. The efolding number for each branch is $\mathcal{N}=\int H_{\text {inf }} d t$, which is given by

$$
\mathcal{N} \simeq \int \frac{d \varphi}{M_{P} \sqrt{\epsilon}}
$$

where $H_{\text {inf }}$ is the scale of inflation in corresponding branch, which is assumed to be nearly unchanged, and $\epsilon$ is the usual slow roll parameter. Here, $\epsilon$ is regarded as constant for simplicity, thus the time spended in corresponding branch is

$$
\Delta t \simeq \frac{1}{H_{\text {inf }}} \frac{\Delta \varphi}{M_{P} \sqrt{\epsilon}} .
$$

Thus in principle, the smaller $\epsilon$ is, the larger this time interval is. We require that the downhill course is not interrupted by the eternal inflation, which corresponds to place a lower limit for $\epsilon$ in each branch, i.e. $\sqrt{\epsilon} \gtrsim \frac{H_{i n f}}{M_{P}}$. Thus we have

$$
\begin{aligned}
\Delta t & \lesssim \frac{1}{H_{\text {inf }}}\left(\frac{\Delta \varphi}{H_{\text {inf }}}\right) \simeq\left(\frac{M_{P}^{2}}{M_{\text {inf }}^{4}}\right) \Delta \varphi \\
& \simeq\left(\frac{M_{P}}{M_{\text {inf }}}\right)^{4} \frac{\Delta \varphi}{M_{P}} t_{P}
\end{aligned}
$$

where $\rho_{\text {inf }}=M_{\text {inf }}^{4}$ is the energy density driving inflation, and $t_{P}=\frac{1}{M_{p}} \simeq \frac{1}{10^{43}} \mathrm{~s}$. When the inflaton potential of each branch has a stringy or supergravity origin, the excursion of effective inflaton in each branch can not exceed $M_{p}$, i.e. $\Delta \varphi \lesssim M_{p}$, we have $\Delta t \lesssim\left(\frac{M_{P}}{M_{i n f}}\right)^{4} t_{P}$. Hence, as is an evident result, we can see that the lower the energy scale of effective potential of a given branch is, the longer the time that the effective inflaton experiences the corresponding branch will be. For example, if $M_{\text {inf }} \sim 10^{15} \mathrm{GeV}, \Delta t \lesssim 10^{-31}$ s can be obtained, of course if $\Delta \varphi>M_{P}$ this time will be longer, while if $M_{\text {inf }} \sim 10^{3} \mathrm{GeV}$, we have $\Delta t \lesssim 10^{21} \mathrm{~s}$, which is actually larger than the age $10^{18} \mathrm{~s}$ of our universe. The time that the effective inflaton experiences a given path should be the sum of the time that it experiences each branch of the corresponding path. Thus if there are some universes which can enter into some branches of low scale inflation, in which $M_{\text {inf }} \lesssim 10^{3} \mathrm{GeV}$, then at present they could be still inflating. This indicates that in this multiverse scenario, due to the diversity of the paths in the field space, it is possible that not all universe have thermalised, even if some universes have thermalised for a long time.

\section{OBSERVATIONS OF A GIVEN OBSERVER AT LATE TIME}

We will show possible observations of a given observer at late time in certain thermalised universes in this multiverse scenario.

\section{A. On primordial perturbation}

The amplitude of perturbation produced around the fork is determined by the character of potential around this point. In general, in a given path that appearance of new branches implies $\frac{\partial^{2} V}{\partial \varphi_{i}^{2}}<0$ at corresponding fork point (while it is positive before this fork point) where $\varphi_{i}$ denotes the effective field along the $i$ th branch. Thus we consider the potential as ${ }^{6}$

$$
V(\varphi)=V_{*}\left(1-\beta \frac{\varphi^{2}}{M_{P}^{2}}\right)
$$

for a detailed analysis, where $\beta$ is a positive and dimensionless parameter. This potential is the same as that of 'hilltop inflation' [30], 31], in which inflation takes place near a maximum of the potential, thus the perturbations generated during inflation can be suitable for observable universe. Here, we mainly concern the perturbation modes produced around the fork point, which may have distinct features and can enter into the horizon of a given observer at late time.

The parent universe is separated into different regions, or baby universes, at the fork point 'A', some of which will be dominated by the effective inflaton along the $\varphi$ direction. The effective mass around the fork point, i.e. $\varphi \simeq 0$, along $\varphi$ direction is $\left|m_{\varphi}^{2}\right|=\frac{2 \beta V_{*}}{M_{P}^{2}}$. The slow roll parameter $\eta$, which together with $\epsilon$ is generally used to depict the slow rolling required by inflation models, is given by $|\eta|=\frac{\left|m_{\varphi}^{2}\right|}{3 H_{\text {inf }}^{2}} \sim \beta$. In general, the amplitude of

\footnotetext{
${ }^{6}$ In principle, for each branch there may be several or lots of fields participating the slow rolling, which leads to assisted inflation [32], see also [33], [34], 35], and also Nflation [36]. Recently, there have been large number of studies on Nflation [37, ,38], 39], 40], 41], 42], and also [43] for staggered inflation.
} 
perturbation is $\sim \frac{1}{V_{\varphi}^{\prime}}$. Thus the amplitude seems to be divergent at the fork point 'A', since $V_{\varphi}^{\prime} \simeq 0$. However, as has been mentioned, the field will be generally brought out of this extremum point by its fluctuation $\delta \varphi=\frac{H_{i n f}}{2 \pi}$. Thus during the inflation driven by $\varphi$, the amplitude of perturbation mode initially produced is given by

$$
\mathcal{P}_{\zeta}^{1 / 2} \sim \frac{1}{M_{P}^{3}} \frac{V^{3 / 2}}{V_{\varphi}^{\prime}} \sim \frac{\sqrt{V_{*}}}{\beta M_{P} \varphi_{i n i}} \sim \frac{1}{|\eta|}
$$

where $\varphi_{i n i} \simeq \delta \varphi=\frac{H_{i n f}}{2 \pi}$ has been used. Thus if $|\eta|>1$ around the fork point, which may be obtained by adjusting the parameter $\beta$ of potential (6), $\mathcal{P}_{\zeta}^{1 / 2}<1$ can be obtained ${ }^{7}$.

In general, for the potentials of new branches around the fork point, there can be other alternatives, since only is $\frac{\partial^{2} V}{\partial \varphi_{i}^{2}}<0$ required at corresponding fork point. We, for example, can also consider the potential as

$$
V(\varphi)=V_{*}\left(1-\lambda \frac{\varphi^{3}}{M_{P}^{3}}\right),
$$

where $\lambda$ is a positive and dimensionless parameter. The effective mass around $\varphi \simeq 0$ is $\left|m_{\varphi}^{2}\right|=\frac{6 \lambda \varphi V_{*}}{M_{P}^{3}}$, which seems smaller than that of the potential (6), since $\varphi$ is smaller. The amplitude of mode initially produced during the inflation along this branch is given by $\mathcal{P}_{\zeta}^{1 / 2} \sim$ $\left.\frac{1}{M_{P}^{3}} \frac{V^{3 / 2}}{V_{\varphi}^{\prime}}\right|_{i n i}$, which is

$$
\mathcal{P}_{\zeta}^{1 / 2} \sim \frac{\sqrt{V_{*}}}{\lambda \varphi^{2}} \sim \frac{\sqrt{V_{*}}}{|\eta| M_{P} \varphi_{i n i}} \simeq \frac{1}{|\eta|},
$$

where $|\eta|=\frac{\left|m_{\varphi}^{2}\right|}{3 H_{i n f}^{2}} \sim \frac{\lambda \varphi}{M_{P}}$ and $\varphi_{i n i} \simeq \delta \varphi=\frac{H_{i n f}}{2 \pi}$ have been used. Thus the case is similar to that for the potential (6). However, if we require $|\eta| \gg 1$, the potential (9) seems to need a larger fine tunning, since $|\eta| \sim \lambda \varphi / M_{p} \gg 1$ means initially $\lambda \gg \frac{M_{p}}{\varphi_{\text {ini }}} \sim \frac{M_{p}}{H_{\text {inf }}}$, which is certainly unnatural. However, in principle we can naturally obtain $|\eta|>1$ by considering other potential, for example, the potential likes $V_{*}\left(1+\xi \ln \frac{\varphi}{\varphi_{*}}\right)$, where

7 The result of (7) is obtained in the slow roll approximation. However, if $H_{\text {inf }}$ is regarded as constant, the amplitude of perturbation can be exactly generated independent of $|\eta| \ll 1$. In this case, actually the evolution of $\varphi$ can be exactly solved, e.g. [20]. The amplitude of curvature perturbations around the fork point is given by

$$
\mathcal{P}_{\zeta}^{1 / 2} \equiv \delta \mathcal{N} \sim \frac{\delta \varphi}{s \varphi} \sim \frac{1}{s}
$$

where $s=1.5\left(\sqrt{\frac{4|\eta|}{3}+1}-1\right)$, and $\varphi \simeq \delta \varphi=\frac{H_{i n f}}{2 \pi}$ has been used, and $\mathcal{N}$ is the efolding number. Therefore, when $|\eta| \ll 1$, $\mathcal{P}_{\zeta}^{1 / 2} \sim 1 /|\eta|$ is consistent with Eq. (7); while for $|\eta| \gtrsim 1, \mathcal{P}_{\zeta}^{1 / 2} \sim$ $1 / \sqrt{|\eta|}$. $\xi$ is a positive and dimensionless constant and $\varphi_{*}$ is certain value at which the fork point begins. The field $\varphi$ will roll down along the direction of $\varphi<\varphi_{*}$ for this potential. The effective mass at about $\varphi \simeq \varphi_{*}$ is $\left|m_{\varphi}^{2}\right|=\frac{\xi V_{*}}{\varphi^{2}}$. Thus $|\eta|=\frac{\left|m_{\varphi}^{2}\right|}{3 H_{\text {inf }}^{2}} \sim \frac{\xi M_{P}^{2}}{\varphi_{\text {ini }}^{2}}$. In general, $\varphi_{\text {ini }} \simeq \varphi_{*}+\delta \varphi=\varphi_{*}$ can be smaller than $M_{P}$. Thus if $\xi$ is not too small, initially $|\eta|>1$ can be obtained. The amplitude of mode initially produced during the inflation along this branch is also the same as (7) and (10), which is given by

$$
\mathcal{P}_{\zeta}^{1 / 2} \sim \frac{\sqrt{V_{*}} \varphi_{i n i}}{\xi M_{P}^{3}} \sim \frac{\sqrt{V_{*}}}{|\eta| M_{P} \varphi_{i n i}} \simeq \frac{1}{|\eta|} .
$$

Thus in principle, along the directions of new branches around the fork point, it seems that both $|\eta| \lesssim 1$ and $|\eta|>1$ are possible, and which one is actually obtained is dependent on the details of the effective potential around the corresponding fork point.

We can see that for $|\eta| \lesssim 1$, the amplitude of perturbation is generally larger than 1 . This means that if the corresponding perturbation mode enters into the horizon at late time, it will inevitably cause gravitational collapse of observable universe, which will rapidly gulp the observer inside a black hole ${ }^{8}$. Thus in this case, it seems the observer can hardly probe the information around the fork point ${ }^{9}$. While for $|\eta| \gg 1$, the case is different, since the amplitude of corresponding perturbation mode is smaller than 1 , and the observer might be able to learn more about the fork point.

When $|\eta| \gg 1$, the potential along the $\varphi$ direction around the fork point should be quite abrupt. Thus in this case the $\varphi$ field will rapidly rolling down along its potential. However, we have required that in each branch there is a region suitable for a period of slow roll inflation. Thus for such case, it seems possible that after a period of fast rolling, the effective field will enter into a period of slow rolling, which drives the inflation of corresponding universe. However, even if there is such a region suitable for slow rolling in corresponding branch, it is also uncertain whether inflation will occur in this branch.

We can simply discuss it as follows. We assume that the potential along $\varphi$ direction around $\varphi \simeq 0$ is step-like, the hight of step is denoted by $\Delta V$ and the following flat region suitable for slow roll inflation is approximately constant denoted by $V_{*}$. When $\Delta V \gg V_{*}$, after the field switches to this branch, the corresponding new universe

\footnotetext{
8 In hybrid inflation, this will lead to the formation of massive primordial black holes in the primordial universe [20], and similar case would also happen in locked inflation, e.g. [44]. The problems of locked inflation has been discussed detailed in [45], which, however, can be avoided in 'old' locked inflation [12].

9 This case is similar to that around the boundary of slow roll eternal inflation, at which $\mathcal{P}_{\zeta} \sim 1$, which in certain sense means that the information of slow roll eternal inflation seems not accessible to a given observer at late time, e.g. [46], and also [47], [48] for the case with large number of fields.
} 
will rapidly become dominated by the kinetic energy of $\varphi$ field, since approximately $\dot{\varphi}_{\text {flat }}^{2} \simeq \Delta V \gg V_{*}$, where the subscript ' $f$ lat' denotes the quantity just entering into the flat region. During the period of kinetic domination, $\dot{\varphi} \sim 1 / a^{3} \sim 1 / t$ can be obtained. Thus during this period the moving distance of $\varphi$ is given by

$$
\begin{aligned}
\Delta \varphi & \simeq \frac{\dot{\varphi}_{\text {flat }}}{H_{\text {inf }}} \int \frac{d t}{t} \simeq \frac{\dot{\varphi}_{\text {flat }}}{H_{\text {inf }}} \ln \sqrt{\frac{\Delta V}{V_{*}}} \\
& \simeq\left(\sqrt{\frac{\Delta V}{V_{*}}} \ln \sqrt{\frac{\Delta V}{V_{*}}}\right) M_{P},
\end{aligned}
$$

where $t_{\text {flat }}=\frac{1}{H_{\text {inf }}}$ and $H_{\text {inf }}^{2} \simeq \frac{V_{*}}{M_{P}^{2}}$ have been used. Thus we can see that if $\Delta V \gg V_{*}$, generally we have $\Delta \varphi \gtrsim M_{P}$. This means that only after $\varphi$ fast rolls to certain value larger than $M_{P}$ might the slow roll inflation occur in this branch. However, if $\Delta \varphi \lesssim M_{P}$, as has been mentioned that if the inflation potential in corresponding branch has a stringy or supergravity origin, the inflation will certainly not occur in this corresponding branch, even if there is a flat region suitable for slow rolling in this branch ${ }^{10}$. However, it is possible that the slow roll inflation might occur at following branch after the effective inflaton passes next fork point. Whereas it is visible that if $\Delta \varphi<M_{P}, \Delta V \lesssim V_{*}$ must be required. $\Delta V \lesssim V_{*}$ means $\dot{\varphi}_{\text {flat }}^{2} \simeq \Delta V \lesssim V_{*}$, thus after the field switches to the corresponding branch, the corresponding new universe will be still inflating. In this case, we have $\dot{\varphi} \sim e^{-3 H_{\text {inf }} t}$. Thus $\Delta \varphi$ is given by

$$
\begin{aligned}
\Delta \varphi & \simeq \dot{\varphi}_{\text {flat }} \int \frac{d t}{e^{3 H_{\text {inf }}}} \simeq \frac{\dot{\varphi}_{\text {flat }}}{H_{\text {inf }}} \frac{1}{e^{3 H_{\text {inf } t_{\text {flat }}}}} \\
& <0.1\left(\sqrt{\frac{\Delta V}{V_{*}}}\right) M_{P},
\end{aligned}
$$

where $t_{\text {flat }}=\frac{1}{H_{\text {inf }}}$ has been used, and the prefactor is $1 /\left(3 e^{3}\right)$, which is smaller than 0.1 . Hence we can see that if $\Delta V \lesssim V_{*}$, then generally $\Delta \varphi \ll M_{P}$. This result is expectant. Thus for $|\eta| \gtrsim 1$, though there is a period of fast rolling of $\varphi$ after the fork point, if the effective potential of corresponding branch is flat enough, the inflation will also occur. In this case, if $\Delta V \gg V_{*}$, the slow roll inflation may occur at following branch after next fork point, while if $\Delta V \lesssim V_{*}$, it can occur at current branch.

Therefore, for the observer be able to live at enough late time, if $|\eta| \ll 1$ around the fork point, he will be rapidly gulfed by a black hole with the scale of his current horizon, since the amplitude $\mathcal{P}_{\zeta} \gtrsim 1$. While $|\eta| \gg 1$, he will see the lower cosmic microwave back-

10 However, if there is an instant production of particles, e.g. 49], [50], the case will be altered. The backreaction of produced particles would induce a stage of trapped inflation [51], 52] or just slow the effective field down by extracting energy from it. ground anisotropies on large angular scales ${ }^{11}$, since there is generally a period of fast rolling preceding the slow roll inflation ${ }^{12}$, e.g. [56], and the bispectrum of fast rolling has been discussed in [55]. When the case is the latter, with the continuously growing of past light cone of the observer, the domain walls will eventually enter into his horizon ${ }^{13}$.

\section{B. On domain wall}

When adjacent $\frac{1}{H_{\text {inf }}}$ regions enter into different branches, the domain wall will inevitably appear between them ${ }^{14}$. However, this actually occurs only when the adjacent regions or universes have thermalized, or the wall will move towards the regions with larger potential energy density, and its moving velocity is the same as that of past light cone of the observer, thus it is impossible that the walls enter into the horizon of a given observer. Here, however, we will consider the case that all adjacent regions or universes have thermalized.

In general, for domain wall, the potential is generally regarded as $\lambda\left(\varphi^{2}-\varphi_{*}^{2}\right)^{2}$, which actually corresponds to the expansion of the potential (6) around the fork point, where $\varphi_{*}=\sqrt{\frac{2}{\beta}} M_{P}$ and $\lambda=\frac{4 V_{*} \beta^{2}}{M_{P}^{4}}$. In some region of space the value of field is $\varphi_{*}$, while in adjacent region it is minus $\varphi_{*}$, so there must be a region where $\varphi=$ 0 , which corresponds to the domain wall. The surface energy density of domain wall is generally given by $\mathcal{E} \sim$ $\frac{\varphi_{*}^{2}}{l}+\lambda \varphi_{*}^{4}$ l, e.g. [64], which is the sum of the contributions of the gradient term and potential energy term, and $l$ is the thickness of the wall. The balance between both terms gives $l \sim \frac{1}{\sqrt{\lambda} \varphi_{*}}$ and $\mathcal{E} \sim \sqrt{\lambda} \varphi_{*}^{3}$.

11 This, for current observations, must occur at corresponding time such that the corresponding scale is near the low multipole of the CMB, or we either would not observe this effect or it has been ruled out.

12 It is also possible that there will be an effective potential with lots of small steps which would not lead to a fast roll and thus a lower amplitude of power spectrum, but instead lead to a modulated power spectrum and bispectrum, e.g. 53] for effects on the power spectrum and [54], 55] for bispectrum, which might be a better probe than a period of fast rolling for this scenario.

13 In general, the period of inflation before thermalization inflates the domain walls away. However, the time that slow roll inflation lasts is finite, thus it is always possible that at enough late time, i.e.the efolding number required by late time evolution is equal to or larger than that given by slow roll inflation. In this sense, one could see the domain walls, even if they are unseen at present epoch.

14 The domain walls may actually contribute and affect the curvature perturbation on large angular scales, which in some sense is similar to that of the bubble wall discussed in [57], [58], [59], 60], 61] for open inflation [62], 63]. The complete investigation on this issue is already beyond our scope. However, it should be pointed out that the behaviors of perturbation spectrum discussed here are not altered qualitatively. 
When the thickness of the wall $l>\frac{1}{H_{\text {inf }}}$, the interior of wall will satisfy the conditions for inflation, thus the topological defect inflation will inevitably occur [65, 66]. For the potential (6), $l>\frac{1}{H_{\text {inf }}}$ implies

$$
\begin{aligned}
& \frac{1}{\sqrt{\lambda} \varphi_{*}} \sim \sqrt{\frac{M_{P}^{4}}{V_{*} \beta^{2}}} \frac{\sqrt{\beta}}{M_{P}}>\frac{1}{H_{\text {inf }}} \sim \frac{M_{P}}{\sqrt{V_{*}}} \\
& \Longrightarrow \quad \beta<1,
\end{aligned}
$$

which means $|\eta|<1$. The topological defect inflation is actually eternal [65, [66], i.e. the inflation of wall will continue eternally, even if the $\varphi$ field in some regions of defect core $\varphi \simeq 0$ has rolled down along the potential and entered into the corresponding branches. This is consistent with the result of (77), where for $|\eta|<1$ around the fork point the amplitude of perturbation mode produced is $\mathcal{P}_{\zeta}>1$. This is because the corresponding mode is actually produced at the boundary of defect eternal inflation, thus its amplitude $\mathcal{P}_{\zeta}>1$ is expectant. Thus in this case even if the past light cone of the observer is enough large, he would have been engulfed inside a black hole, before he has the opportunity to see the wall.

However, if $|\eta| \gg 1$, the domain wall will not inflate. Thus it is possible that at late time the domain wall will enter into the horizon of a given observer. The domain walls are produced during the evolution of field around the fork point. The length scale of domain wall is initially about $\frac{1}{H_{\text {inf }}}$, and then is brought to $R \simeq \frac{1}{H_{\text {inf }}} e^{\mathcal{N}}$ by inflation in corresponding branch. Hereafter, it is stretched by the expansion of standard cosmology. Thus given that the domain wall enters into the horizon at present $R \sim \frac{1}{H_{0}}$, its mass can be estimated as, e.g. [67], 66],

$$
\begin{aligned}
M_{\text {wall }} & \sim \frac{\mathcal{E}}{H_{0}^{2}} \sim 10^{65} \sqrt{\lambda}\left(\frac{\varphi_{*}}{100 \mathrm{Gev}}\right)^{3} \mathrm{~g} \\
& \sim 10^{10} \sqrt{\lambda}\left(\frac{\varphi_{*}}{100 \mathrm{Gev}}\right)^{3} M_{H_{0}},
\end{aligned}
$$

where $M_{H_{0}} \sim \frac{\rho_{0}}{H_{0}^{3}}$ is the mass of observable universe at present, and $\rho_{0}$ is the energy density of observable universe. Thus unless $\lambda$ and $\varphi_{*}$ are unacceptablely small, $M_{\text {wall }}$ is generally larger than $M_{H_{0}}$. Thus when the domain wall enters into the horizon of a given observer at late time, it will lead to a large fluctuation of energy density in corresponding regions. Here, relevant phenomena might be significant, which will be left for future works.

\section{CONCLUSION}

In a given path with multiple branches, each of which may drive a period of slow roll inflation, it can be ex- pected that there are some fork points, at which one branch is bifurcated into different branches, or many branches converge into one or several branches. In this paper, it is showed that if there is a web of such branches in a given field space, a multiverse separated by domain wall network will actually come into being.

Rolling down hill, the effective field will be expected to enter into certain stable or metastable minimum, which corresponds to the terminal of a given path. In principle, there should be large number of such minima, some of which might be suitable for the world where we live. We estimated the number of universes entering into certain terminal minimum, which is generally quite large. For a given observer be able to live permanently, he might be able to explore the modes produced around the fork point. In this case, if $|\eta| \ll 1$ along the direction of effective inflaton around the fork point, he will be gulfed by a black hole with the scale of his current horizon rapidly, while if $|\eta| \gg 1$, he will see the lower cosmic microwave background anisotropies on large angular scales, since there is generally a period of fast rolling preceding the slow roll inflation. When the case is the latter, at enough late time, the domain walls will possibly enter into the horizon of this given observer, which would bring a distinct observation.

In general, the multiverse scenario can be obtained in eternal inflation, which is based on the random walk of inflaton field induced by itself large quantum fluctuation. Here, however, the multiverse scenario is implemented by the classical rolling of inflaton along a web of branches of its effective potential which happens at a relatively lower energy scale. In reality to the multiverse scenario, due to the complexity of potential of field space, both effects may be possible to contribute the number of universes, and the possibility that a region of space evolving along one branch classically tunnels to another close-by branch is also not excluded. Thus the exploration of relevant issues will be interesting. In addition, it is also significant to investigate a possible implement of such a scenario in string theory, which will be studied in the future.

Acknowledgments We thank T. Battefeld, Y.F. Cai, Y. Wang for discussions. This work is supported in part by NSFC under Grant No: 10775180, in part by the Scientific Research Fund of GUCAS, in part by CAS under Grant No: KJCX3-SYW-N2, in part by the Ministry of Science and Technology of China under Grant No:2010CB832800.
[1] S. Kachru, R. Kallosh, A. Linde, S.P. Trivedi, Phys. Rev. D68, 046005 (2003).
[2] L. Susskind, arXiv:hep-th/0302219. 
[3] A.H. Guth, Phys. Rev. D23, 347 (1981).

[4] A.D. Linde, Phys. Lett. B108, 389 (1982).

[5] A. Albrecht, P.J. Steinhardt, Phys. Rev. Lett. 48, 1220 (1982).

[6] A.A. Starobinsky, JETP Lett. 30, 682 (1979)

[7] D. Polarski, A.A. Starobinsky, Nucl. Phys. B385, 623 (1992).

[8] B. Feng, X.M. Zhang, Phys. Lett. B570, 145 (2003).

[9] G. Dvali, S. Kachru, arXiv:hep-th/0309095.

[10] R. Easther, arXiv:hep-th/0407042.

[11] C.P. Burgess, R. Easther, A. Mazumdar, D.F. Mota, T. Multamaki, JHEP 0505, 067 (2005).

[12] Y. Liu, Y.S. Piao, Z.G. Si, JCAP 0905, 008 (2009).

[13] A.D. Linde, Phys. Lett. B259, 38 (1991); Phys. Rev. D49, 748 (1994).

[14] F.C. Adams, K. Freese, Phys. Rev. D43, 353 (1991).

[15] A.R Liddle, D.H Lyth, Phys. Rept. 231, 1 (1993).

[16] E.J. Copeland, A.R. Liddle, D.H. Lyth, E.D. Stewart, D. Wands, Phys. Rev. D49, 6410 (1994).

[17] E.D. Stewart, Phys. Lett. B345, 414 (1995).

[18] G. Lazarides, C. Panagiotakopoulos, Phys. Rev. D52, 559 (1995).

[19] G. Lazarides, Q. Shafi, Phys. Lett. B372, 20 (1996).

[20] J. Garcia-Bellido, A. Linde, D. Wands, Phys. Rev. D54, 6040 (1996).

[21] A. Vilenkin, Phys. Rev. D27, 2848 (1983).

[22] A. Linde, Phys. Lett. B175, 395 (1986).

[23] P.J. Steinhardt, in "The Very Early Universe", ed. by G.W. Gibbons, S.W. Hawking and S.T.C. Siklos (Cambridge University Press, 1983).

[24] A.H. Guth, E.J. Weinberg, Nucl. Phys. B212, 321 (1983).

[25] A.A. Starobinsky, in "Current Topics in Field Theory, Quantum Gravity and Strings," edited by H.J. de Vega and N. Sanchez, Lecture Notes in Physics, Vol.26 (Springer, Heidelberg, 1986), 107.

[26] A.S. Goncharov, A.D. Linde, V.F. Mukhanov, Int. J. Mod. Phys. A2, 561 (1987).

[27] A.D. Linde, Nucl. Phys. B372, 421 (1992).

[28] A.D. Linde, "Particle Physics and Inflationary Cosmology", (Harwood, Chur, Switzerland, 1990); Contemp. Concepts Phys. 5, 1 (2005), arXiv:hep-th/0503203.

[29] M. Li, Y. Wang, arXiv:0903.2123

[30] L. Boubekeur, D.H. Lyth, JCAP 0507, 010 (2005).

[31] K. Kohri, C.M. Lin, D.H. Lyth, JCAP 0712, 004 (2007).

[32] A.R. Liddle, A. Mazumdar, F.E. Schunck, Phys. Rev. D58, 061301 (1998).

[33] A. Mazumdar, S. Panda, A. Perez-Lorenzana, Nucl. Phys. B614, 101 (2001).

[34] Y.S. Piao, R.G. Cai, X.M. Zhang, Y.Z. Zhang, Phys. Rev. D66, 121301 (2002).

[35] M. Majumdar, A.C. Davis, Phys. Rev. D69, 103504 (2004).

[36] S. Dimopoulos, S. Kachru, J. McGreevy, J.G. Wacker, JCAP 0808, 003 (2008).

[37] L. Alabidi, D. Lyth, JCAP 0605, 016 (2006).
[38] R. Easther, L. McAllister, JCAP 0605, 018 (2006).

[39] S.A Kim, A.R. Liddle, Phys. Rev. D74, 023513 (2006); Phys. Rev. D76, 063515 (2007).

[40] Y.S. Piao, Phys. Rev. D74, 047302 (2006); I. Ahmad, Y.S. Piao, C.F. Qiao, JCAP 0802, 002 (2008).

[41] S. Thomas, J. Ward, Phys. Rev. D76, 023509 (2007); J. Ward, JHEP 0712, 045 (2007).

[42] Y.F. Cai, W. Xue, arXiv:0809.4134; Y.F. Cai, H.Y. Xia, arXiv:0904.0062

[43] D. Battefeld, T. Battefeld, A.C. Davis, JCAP 0810, 032 (2008); D. Battefeld, T. Battefeld, JCAP 0903, 027 (2009).

[44] R. Easther, J. Khoury, K. Schalm, JCAP 0406, 006 (2004).

[45] E.J. Copeland, A. Rajantie, JCAP 0502, 008 (2005).

[46] R. Bousso, B. Freivogel, I.S. Yang, Phys. Rev. D74, 103516 (2006).

[47] I. Ahmad, Y.S. Piao, C.F. Qiao, Phys. Lett. B673, 1 (2009).

[48] P. Adshead, R. Easther, E.A. Lim, Phys. Rev. D79, 063504 (2009).

[49] G. Felder, L. Kofman, A. Linde, Phys. Rev. D59, 123523 (1999).

[50] G. Felder, L. Kofman, A. Linde, Phys. Rev. D60, 103505 (1999).

[51] L. Kofman, A. Linde, X. Liu, A. Maloney, L. McAllister, E. Silverstein, JHEP 0405, 030 (2004).

[52] D. Green, B. Horn, L. Senatore, E. Silverstein, arXiv:0902.1006

[53] J. Adams, B. Cresswell, R. Easther, Phys. Rev. D64, 123514 (2001).

[54] X. Chen, R. Easther, E.A. Lim, JCAP 0706, 023 (2007).

[55] X. Chen, R. Easther, E.A. Lim, JCAP 0804, 010 (2008).

[56] C.R. Contaldi, M. Peloso, L. Kofman, A. Linde, JCAP 0307002 (2003).

[57] J. Garriga, Phys. Rev. D54, 4764 (1996).

[58] M. Sasaki, T. Tanaka, Phys. Rev. D54, 4705 (1996).

[59] J. Garriga, X. Montes, M. Sasaki, T. Tanaka, Nucl. Phys. B513, 343 (1998); Nucl. Phys. B551, 317 (1999).

[60] J. Garcia-Bellido, J. Garriga and X. Montes, Phys. Rev. D57, 4669 (1998); Phys. Rev. D60, 083501 (1999).

[61] A.D. Linde, M. Sasaki, T. Tanaka, Phys. Rev. D59, 123522 (1999).

[62] M. Bucher, A.S. Goldhaber, N. Turok, Phys. Rev. D52, 3314 (1995).

[63] K. Yamamoto, M. Sasaki, T. Tanaka, Astrophys. J. 455, 412 (1995).

[64] A. Vilenkin, Phys. Rep. 121, 263 (1985).

[65] A. Linde, Phys. Lett. B327, 208 (1994).

[66] A. Vilenkin, Phys. Rev. Lett. 72, 3137 (1994).

[67] E.W. Kolb, M.S. Turner, "The Early Universe", (Addison-Wesley Publising Company, 1990).

[68] V. Mukhanov, "Physical Foundations of Cosmology", (Cambridge University Press, 2005). 